Die in das Gesundheitswesen eingebundene ärztliche Weiterbildung steht zunehmend unter finanziellem Druck und muss effizienter gestaltet werden. Internationale Erfahrungen zeigen, dass Assessmentinstrumente helfen, Lernprozesse zu strukturieren und zu fördern. Im Rahmen eines Pilotprojekts wurde in der Schweiz seit Ende 2006 an 18 Kliniken das Arbeitsplatz-basierte Assess- ment ( $A b A)$ eingesetzt. Die daraus gewonnenen Resultate und Erfahrungen zeigen auf, dass AbA auch in der Schweiz in verschiedenen Disziplinen anwendbar ist. Die Feedback- und die Kommunikationskultur erfährt dadurch eine stärkere Gewichtung, was zur Verbesserung der Qualität der ärztlichen Weiterbildung und der Patientensicherheit beiträgt.

\title{
Drei Jahre Erfahrung mit Arbeitsplatz- basiertem Assessment (Mini-CEX und DOPS) in der ärztlichen Weiterbildung
}

\author{
Stephanie Montagnea, \\ Patrick Jucker-Kupper ${ }^{a}$, \\ Christoph Berendonk ${ }^{a}$, \\ Anja Rogausch , \\ Christine Beyelera, \\ Max Giger ${ }^{b}$ sowie die Leiter \\ und Projektverantwortlichen \\ der beteiligten Weiter- \\ bildungsstätten ${ }^{c}$
}

a Abteilung für Assessment und Evaluation (AAE) Institut für Medizinische Lehre (IML), Universität Bern

b Schweizerisches Institut für ärztliche Weiter- und Fortbildung (SIWF), Bern

c Eine Namensliste befindet sich am Ende des Artikels.

1 Ursprünglich unter dem Titel «Strukturiertes Feedback in der ärztlichen Weiterbildung: Mini-CEX und DOPS».

Korrespondenz:

Arbeitsplatz-basiertes Assessment

Universität Bern

Institut für Medizinische Lehre Abteilung für Assessment und Evaluation Konsumstrasse 13 CH-3010 Bern

aba@iml.unibe.ch

www.iml.unibe.ch/aba

\section{Einleitung}

Die ärztliche Weiterbildung bereitet auf die selbständige Berufsausübung in einem bestimmten Fachgebiet vor und erfolgt grösstenteils in Spitälern. Diese sind sowohl Weiterbildungsstätten als auch Dienstleistungsbetriebe. Als solche sehen sie sich einem stetig wachsenden (Leistungs-)Druck ausgesetzt. Die ärztliche Weiterbildung gerät im Spannungsfeld von effizienter Patientenversorgung, Umsetzung des Arbeitsgesetzes, begrenzten Arbeitskräften und zunehmenden administrativen Aufgaben unter massiven Druck. Dies führt zur Beschränkung von Weiterbildungsstellen und gefährdet die Qualität der Weiterbildung. Diesen Tendenzen kann durch Effizienzsteigerung der Weiterbildung entgegengewirkt werden.

Mit dieser Zielsetzung wurde Ende 2006 in der Schweiz an mehreren Kliniken verschiedener Fachrichtungen das Pilotprojekt «Arbeitsplatz-basiertes Assessment» $(\mathrm{AbA})^{1}$ lanciert [1]. Das Projekt wird vom Schweizerischen Institut für ärztliche Weiter- und Fortbildung (SIWF) finanziert und von der Abteilung für Assessment und Evaluation (AAE) des Instituts für Medizinische Lehre (IML) der Universität Bern umgesetzt und wissenschaftlich begleitet.

Der vorliegende Bericht fasst die bisherigen Erfahrungen an den Pilotkliniken zusammen und zeigt die daraus gewonnenen Erkenntnisse sowie notwendigen Anpassungen auf. Wir stützen uns dabei auf die Auswertung der Assessmentbögen sowie einer begleitenden Evaluation in Form einer Online-Umfrage ${ }^{2}$ bzw. einer Fokusgruppe ${ }^{3}$.

\section{Prinzip des Arbeitsplatz-basierten Assessments}

Die ärztliche Weiterbildung basiert traditionellerweise auf der praktischen Arbeit am und mit dem Patienten unter Supervision sowie auf theoretischem Unterricht. Das Arbeitsplatz-basierte Assessment (AbA) stärkt diese bewährten Elemente, erweitert sie aber gleichzeitig um dasjenige des strukturierten Feedbacks. Die Trias «Be- obachten - Festhalten - Feedback geben» bildet die Grundlage zweier in der medizinischen Aus- und Weiterbildung verbreiteter AbA-Instrumente [2-5], der Mini-Clinical Evaluation Exercise (Mini-CEX) [6] und der Direct Observation of Procedural Skills (DOPS) [7]. Bei beiden Instrumenten steht die unmittelbare Arbeit des Assistenzarztes ${ }^{4}$ mit dem Patienten im Zentrum. Während die Mini-CEX den Fokus auf die Kommunikation mit dem Patienten oder die klinische Untersuchung legt, werden bei der DOPS die manuell-technischen Fertigkeiten ins Zentrum gerückt. Die Vorgehensweise ist bei beiden Instrumenten identisch:

- Der Weiterbildner ${ }^{4}$ beobachtet den Assistenzarzt während 10-15 Minuten bei einer alltäglichen Arzt-Patienten-Interaktion (z. B. Anamnese, Aufklärungsgespräch, Untersuchung, Eingriff).

- Im Anschluss an die Beobachtung beurteilt der Weiterbildner die ärztliche Handlung anhand definierter Kriterien. Der Assistenzarzt schätzt sich anhand derselben Kriterien selbst ein.

- In einem abschliessenden Gespräch von 5-10 Minuten Dauer werden Fremd- und Selbstbeurteilung verglichen, Stärken und Schwächen besprochen sowie die weiteren Lernschritte gemeinsam definiert.

Der systematische Ansatz bringt den Vorteil, dass Anforderungen und Erwartungen explizit formuliert sowie Stand und Ziele der Weiterbildung in regelmässigen Abständen überprüft werden; Weiterbildungslücken können somit effizient geschlossen werden.

Ein einzelnes Assessment entspricht einer Momentaufnahme im klinischen Alltag. Erst mehrere Momentaufnahmen aus diversen Blickwinkeln ergeben ein Gesamtbild. Die Assessments sollten daher wiederholt, in unterschiedlichen Situationen sowie mit wechselnden Weiterbildnern durchgeführt werden.

\section{Erste Erfahrungen ...}

Ende September 2009 waren 18 Kliniken aus acht Fachrichtungen (Innere Medizin, Chirurgie, Gefäss- 
2 An der freiwilligen und anonymen Umfrage Mitte 2008 nahmen 21 Assistenzärzte und 16 Weiterbildner aus 9 Pilotkliniken und 5 Fachrichtungen (Innere Medizin, Chirurgie, Gefässchirurgie, Gynäkologie und Geburtshilfe, Psychiatrie und Psychotherapie, Rheumatologie und Dermatologie) teil.

3 Am 9.9.2009 fand auf Einladung des SIWF und der AAE/IML ein Treffen der Vertreter von Pilotkliniken statt. Die Namensliste finde sich am Ende des Artikels.

4 Die männliche Form gilt in gleicher Weise für das weibliche Geschlecht.

5 Gemäss Art. 20 Weiterbildungsordnung (WBO).

6 An der Medizinischen Fakultät Bern werden MiniCEX und DOPS ab 2010 fester Bestandteil des reformierten Masterstudiengangs sein. chirurgie, Gynäkologie und Geburtshilfe, HNO, Psychiatrie und Psychotherapie, Rheumatologie und Dermatologie) am Pilotprojekt beteiligt. Bis zu diesem Zeitpunkt wurden 443 Mini-CEX und 244 DOPS von 260 Assistenzärzten und 129 Weiterbildnern durchgeführt. Die Beobachtung dauerte im Mittel 15 Minuten, das Feedback 5 Minuten. Das Leistungsniveau entsprach gemäss der Einschätzung der Weiterbildner weitgehend dem erwarteten Weiterbildungsstand der Assistenzärzte (Mittelwert Globalurteil $=6,2$ auf einer Skala von 1-9; Standardabweichung SD = 1,9). Die Selbsteinschätzung der Assistenzärzte fiel dagegen mehrheitlich kritischer aus (Mittelwert 5,4; SD 1,7). Sowohl Assistenzärzte als auch Weiterbildner zeigten sich mit Mini-CEX und DOPS zufrieden (Mittelwert \pm SD: Mini-CEX / DOPS 6,9 $\pm 1,8 / 7,4 \pm 1,8$ bei Assistenzärzten; 7,0 $0 \pm 1,8 / 7,8 \pm 1,5$ bei Weiterbildnern auf einer Skala von 1-10).

\section{Benefit}

Die Hauptbeweggründe für die Teilnahme am Pilotprojekt waren der Wunsch nach einer strukturierten, möglichst objektiven Beurteilung der Assistenzärzte und der Wunsch nach einer Verbesserung der Feedback- und Kommunikationskultur. Mit Einführung des AbAs hat an den Pilotkliniken eine Sensibilisierung und vermehrte Diskussion rund um das Thema Weiterbildung stattgefunden. Mini-CEX und DOPS werden als wertvolle Ergänzung zum (halb-)jährlich stattfindenden Evaluationsgespräch ${ }^{5}$ wahrgenommen. Die strukturierte Beobachtung erlaubt einen vertieften Einblick in die Arbeit der Weiterzubildenden und ermöglicht ein spezifisches, konkretes und zeitnahes Feedback. Eine «Kurskorrektur» innerhalb der Weiterbildungszeit an der betreffenden Weiterbildungsstätte wird dadurch ermöglicht.

Im Gegensatz zu den meisten ärztlichen Tätigkei-

Angst vor Bewertung). Vonseiten der Weiterbildner wurden ungenügende Schulung als Weiterbildner im Allgemeinen und im Erteilen von Feedback im Speziellen, Wahrnehmung des AbAs als Prüfung und die zusätzliche zeitliche Belastung genannt. Von beiden Gruppen wurde insbesondere die unklare Verantwortlichkeit betreffend Terminvereinbarung bemängelt.

\section{... und daraus gewonnene Erkenntnisse}

\section{Potential}

Die Teilnehmer der Fokusgruppe waren sich einig, dass AbA das Potential für einen Kulturwandel mit sich bringt, einen solchen gleichzeitig aber auch benötigt, um effektiv und effizient eingesetzt werden zu können. Die Weiterbildung per se und deren Qualität werden durch AbA aktiv thematisiert. Ärztliches Handeln kann mit AbA evaluiert werden; die Assistenzärzte können aufgrund ihres Kompetenzstandes in der Patientenbetreuung eingesetzt werden. Mit zunehmender Verbreitung des AbAs, besonders auch in der Ausbildung, wird die zukünftige Generation der Weiterzubildenden mit $\mathrm{AbA}$ vertraut sein und regelmässige Feedbacks einfordern. Den Weiterbildungsstätten ermöglicht die vermehrte Strukturierung der Weiterbildung durch regelmässige Assessments einen Wettbewerbsvorteil bei der Rekrutierung von Assistenzärzten.

\section{Notwendige Anpassungen}

Um die Durchführung des AbAs im klinischen Alltag zu erleichtern, plädierten sowohl Assistenzärzte als auch Weiterbildner für mehr Struktur bei der Organisation der Assessments (Festlegung von Zeitfenstern für die Durchführung). Ein Grossteil der Kliniken hat entsprechende Massnahmen getroffen - andere erklärten die Assessments zur Voraussetzung für die Durchführung der Evaluationsgespräche. Gute Erfahrungen wurden gemacht mit einer hohen Frequenz von Assessments; der Prüfungscharakter verliert sich hierbei von selbst, Evaluation und Feedback werden sowohl für Assistenzärzte als auch Weiterbildner zur täglichen Routine. Das Einfordern von Feedback hängt einerseits von den Lernzielen und der Motivation des Assistenzarztes und andererseits von der Art der Supervision des Weiterbildners ab [8]. Die Weiterbildner müssen in Assessment und Feedback geschult werden [9]. Von mehreren Seiten wird eine vermehrte Einbindung des AbAs in ein Gesamtkonzept (Weiterbildungskonzepte der Kliniken, Logbücher, Weiterbildungsprogramme) begrüsst. Im revidierten Weiterbildungsprogramm für Allgemeine Innere Medizin [10] wurde diese Forderung integriert. In den Logbüchern der Weiterbildungsgänge Psychiatrie und Dermatologie hat $\mathrm{AbA}$ ebenfalls Eingang gefunden. zu wenig offensichtlich. Als Haupthindernisse für die Umsetzung des AbAs im klinischen Alltag nannten die Assistenzärzte den grossen Arbeitsanfall, die schwierige Terminkoordination mit den Weiterbildnern, Rollenkonflikte (Hemmung, Zeitressourcen der Weiterbildner zu beanspruchen) sowie ein Unbehagen gegenüber den Assessments (Wahrnehmung als Prüfung;

\section{Schlussfolgerungen}

Die Resultate und Erfahrungen aus unserem Pilotprojekt zeigen, dass AbA auch in der Schweiz durchführbar und in verschiedenen Disziplinen anwendbar ist. Die Feedback- und Kommunikationskultur erfahren 
durch AbA eine stärkere Gewichtung, was zur Verbesserung der Qualität der ärztlichen Weiterbildung und der Patientensicherheit [11] beiträgt.

AbA wurde von den Kliniken grösstenteils positiv aufgenommen - bedarf jedoch einer sorgfältigen Vorbereitung (Schulung) des Teams und - wie jede Neuerung - einer gewissen Adaptationszeit. Erfahrungen aus anderen Ländern zeigen, dass die fehlende Vertrautheit mit AbA nebst organisatorischen Problemen und zeitlichem Aufwand ebenfalls zu den Haupthürden bei der Einführung zählt $[12,13]$. Bei der breiten Umsetzung des AbAs in der ärztlichen Weiterbildung, wie dies das revidierte Weiterbildungsprogramm Allgemeine Innere Medizin vorsieht, soll den von den Pilotkliniken gemachten Erfahrungen Rechnung getragen werden. Fachspezifische Anliegen und Besonderheiten werden vermehrt zu berücksichtigen sein.

Die mit AbA einhergehenden Feedback- und Kommunikationskulturen sind wichtige Stützen einer qualitativ hochwertigen ärztlichen Weiterbildung. AbA trägt dazu bei, dass unter den gegebenen Rahmenbedingungen des Gesundheitswesens heute wie auch in Zukunft eine effiziente und zielgerichtete ärztliche Weiterbildung angeboten werden kann.

\section{Verdankungen}

Wir danken allen Assistenzärzten und Weiterbildnern der am Pilotprojekt beteiligten Kliniken für die Durchführung des AbAs. Ein spezieller Dank geht an die Teilnehmer der Online-Umfrage sowie an die Vertreter der Pilotkliniken, die am Treffen vom 9.9.2009 teilgenommen haben. Zudem danken wir Dr. med. M. Perrig (Klinik für Allgemeine Innere Medizin, Inselspital Bern) und Dr. med. F. Nohl (Medizinische Klinik, Zieglerspital Bern) für den Erfahrungsbericht über $\mathrm{AbA}$ in der medizinischen Ausbildung an der Universität Bern, PD Dr. med. E. Bächli (Medizinische Klinik, Spital Uster) für die Vertretung der Chefärztevereinigung Innere Medizin, Dr. med. C. Kim (Klinik für Viszerale Chirurgie, Inselspital Bern) für das Einbringen der Sichtweise des Geschäftsausschusses VSAO und Prof. Dr. med. W. Langewitz (Psychosomatik, Universitätsspital Basel) für die Moderation der Veranstaltung.

\section{Namensliste der Leiter und Projektverantwort- lichen der beteiligten Weiterbildungsstätten in chronologischer Reihenfolge}

Prof. Dr. med. V. Briner und Dr. med. T. Hodel* (Departement Innere Medizin, Kantonsspital Luzern); Prof. Dr. med. W. Reinhart, Dr. med. T. Wieland und Dr. med. R. Strub (Departement Innere Medizin, Kantonsspital Graubünden); Dr. med. J. Kurmann, Dr. med. M. Kendall und Dr. med. L. Schlegel* (Psychiatrie Luzern); Prof. Dr. med. M. Wolfensberger und PD Dr. med. A. Welge-Lüsse (HNO-Klinik, Universitätsspital Basel); Dr. med. J. Knaus (Chirurgische Klinik, Spital Lachen); Dr. med. M. Widmer* (Klinik für Herz- und Gefässchirurgie, Inselspital Bern); Prof. Dr. med. U. Laffer, Dr. med. H. U. Würsten*, Dr. med. P. M. Sutter (Chirurgische Klinik, Spitalzentrum Biel); Prof. Dr. med. M. Müller (Frauenklinik, Inselspital Bern); Prof. Dr. med. M. Röthlin* (Chirurgische Klinik, Spital Münsterlingen); Prof. Dr. med. S. Stöckli (HNO-Klinik, Kantonsspital St. Gallen); PD Dr. med. F. Salomon (Medizinische Klinik, Spital Lachen); PD Dr. med. L.T. Heuss (Medizinische Klinik, Spital Zollikerberg); Dr. med. F. Maurer-Marti, Dr. med. G. Zimmermann (Frauenklinik, Bürgerspital Solothurn); Dr. med. N. Zerkiebel* (Medizinische Klinik und Rehabilitation, Klinik Susenberg ZH); Dr. med. E. Bonvin, Dr. med. S. Etienne* (Psychiatrische Klinik, Monthey); Prof. Dr. med. P. Villiger und Dr. med. H. Schwarz* (Klinik für Rheumatologie, Inselspital Bern); Prof. Dr. med. P. Itin, Dr. med. P. Häusermann (Dermatologische Klinik, Universitätsspital Basel); PD Dr. med. N. Bürki (Frauenklinik, Kantonsspital Liestal); Dr. med. B. von Castelberg, Dr. med. S. von Orelli (Frauenklinik, Stadtspital Triemli).

\section{Literatur}

1 Berendonk C, Beyeler C, Westkämper R, Giger M. Strukturiertes Feedback in der ärztlichen Weiterbildung: Mini-CEX und DOPS. Schweiz Ärztezeitung. 2008;89(32):1337-40.

2 Davies H, Archer J, Southgate L, Norcini J. Initial evaluation of the first year of the Foundation Assessment Programme. Med Educ. 2009 Jan;43(1):74-81.

3 Scheele F, Teunissen P, Van Luijk S, et al. Introducing competency-based postgraduate medical education in the Netherlands. Med Teach. 2008;30(3):248-53.

4 Kogan JR, Hauer KE. Brief report: Use of the miniclinical evaluation exercise in internal medicine core clerkships. J Gen Intern Med. 2006 May;21(5):501-2.

5 Hatala R, Ainslie M, Kassen BO, Mackie I, Roberts JM. Assessing the mini-Clinical Evaluation Exercise in comparison to a national specialty examination. Med Educ. 2006 Oct;40(10):950-6.

6 Norcini JJ, Blank LL, Arnold GK, Kimball HR. The mini-CEX (clinical evaluation exercise): a preliminary investigation. Ann Intern Med. 1995 Nov 15;123(10):795-9.

7 Wragg A, Wade W, Fuller G, Cowan G, Mills P. Assessing the performance of specialist registrars. Clin Med. 2003 Mar-Apr;3(2):131-4.

8 Teunissen PW, Stapel DA, van der Vleuten C, Scherpbier A, Boor K, Scheele F. Who wants feedback? An investigation of the variables influencing residents' feedback-seeking behavior in relation to night shifts. Acad Med. 2009 Jul;84(7):910-7.

9 PMETB. National Training Surveys 2008-2009 Key findings. www.pmetb.org.uk.Chapter 3: Workplace Based Assessment:37-47.

10 Giger M. Das neue Weiterbildungsprogramm für Allgemeine Innere Medizin: Stärkung der Hausarztmedizin. Schweiz Ärztezeitung. 2009;90(50):1951-2.

11 Mazzocco K, Petitti DB, Fong KT et al. Surgical team behaviors and patient outcomes. American journal of surgery. 2009 May;197(5):678-85.

12 Johnson G, Barrett J, Jones M, Parry D, Wade W. Feedback from educational supervisors and trainees on the implementation of curricula and the assessment system for core medical training. Clin Med. 2008 Oct;8(5):484-9.

13 PMETB. Workplace Based Assessment - A Guide for Implementation. www.pmetb.org.uk. 2009. die am Treffen vom 9.9.2009 teilgenommen haben. 